\title{
Modified Rancimat Method for Evaluation of Antioxidative Effect against Skin Lipids
}

\author{
Hye Yoon Park, Ho Sik Rho, ${ }^{*}$ Duck Hee Kim, Han Kon Kim, Quan-Quan He, ${ }^{\dagger}$ and Jaeho Yeon ${ }^{\dagger}$ \\ AmorePacific R\&D Center in Yongin, Korea. *E-mail: thiocarbon@freechal.com \\ ${ }^{\dagger}$ AmorePacific R\&D Center in Shanghai, China \\ Received March 2, 2010, Accepted April 12, 2010
}

Key Words: Antioxidative effect, Modified rancimat method, Artificial skin lipid, meso-Dihydroguaiaretic acid

Skin is a highly complicated tissue which possesses the largest surface area in the body and serves as the protective layer for internal organs. It is designed to give both physical and biochemical protection, and is equipped with a large number of defense mechanisms. ${ }^{1}$ However, skin is very susceptible to oxidative reaction, because there are rich contents of lipids, proteins and DNA in the skin cell, all of which are very sensitive to the oxidation process. ${ }^{2}$ Lipid peroxidation could be induced from excessive exposure to UV light, and has been considered as a major factor for skin aging processes. ${ }^{3}$ In particular, squalene, main component of skin surface polyunsaturated lipids, is easily peroxidized. ${ }^{4}$ The initial product of peroxidized squalene, squalene-monohydroperoxide $(\mathrm{Sq}-\mathrm{OOH})$, is produced at the human skin by natural exposure to sun light. Recently, the damages of hairless mice skin by the topical application of $\mathrm{Sq}-\mathrm{OOH}^{5}$ have been reported. Repeated application of $\mathrm{Sq}-\mathrm{OOH}$ to the hairless mice induced clear skin wrinkles. Thus, in order to prevent or retard skin aging process, prevention of skin lipid peroxidation by natural or chemical antioxidants is desirable. To identify efficient antioxidants in cosmetic applications, a convenient assay method suitable for the estimation of skin lipid peroxidation is necessary. Rancimat ${ }^{6}$ is a convenient method which can evaluate the lipid peroxidation by observing the change of electrical conductivity due to the formation of volatile dicarboxylic acids. It has been used in food industry to determine the peroxidation of unsaturated fatty acid components. However, the rancimat method is not suited for skin research, because the composition of oils used as a lipid source in this method is very different from that of skin lipid. ${ }^{6 c}$ In this report, we demonstrate that modified rancimat method is a powerful tool for screening natural antioxidants for skin lipid peroxidation. We changed composition of lipid source to what is very close to skin lipid.

At first, we tested antioxidative activities of several natural

Table 1. Induction period and $P f$ value by general rancimat method

\begin{tabular}{ccc}
\hline Compounds & Induction period $(\mathrm{h})^{a}$ & $P f^{b}$ \\
\hline Control & 14.7 & 1.0 \\
BHT & 58.6 & 4.0 \\
SBG & 24.0 & 1.6 \\
POL & 19.0 & 1.3 \\
RCN & 17.8 & 1.2 \\
\hline
\end{tabular}

${ }^{a}$ Values are given as means of three experiments. ${ }^{b} P f$ (protection factor $)=$ Induction period of antioxidant/Induction period of control. extracts by using general rancimat method. As shown in Table 1, extracts from Korean medicinal plants presented different induction time on retarding the peroxidation of corn oil. Their activities were compared with BHT as a positive control. Among the three natural extracts, Scutellaria baicalensis Georgi (SBG) extract was found to have the most powerful antioxidative activity. Portulaca oleracea L. (POL) extracts appeared to be the compound with the next activity and Rheum coreanum Nakai $(\mathrm{RCN})$ extract was less active than other extracts. The order of activity is $\mathrm{BHT}>\mathrm{SBG}>\mathrm{POL}>\mathrm{RCN}$. However, ranges of $P f$ values of tested natural product are too small (from 1.2 to 1.6). Thus, sensitivity is not enough for primary screening of antioxidative activity.

To establish the modified rancimat system suitable for skin lipid peroxidation studies, we prepared the artificial skin lipid and used it instead of corn oil. The prepared artificial skin lipid ${ }^{7}$ was consisted of pork lard (33\%), stearic acid (24\%), lanolin (22\%), squalene (12\%) and cholesterol (4\%). This composition is similar to that of human skin. According to the Table 2, the results of new rancimat method were different from previous general rancimat data. Surprisingly, SBG extract shows the strongest antioxidative activity among the tested samples. Induction period of SBG was 11.4 times longer than that of control with this new method. The sensitivity for evaluation of antioxidative activity was dramatically increased due to the wide ranges of $P f$ value (from 2.2 to 11.4). It is interesting to observe that the order of activity between RCN and ROL was reversed. In contrast to previous rancimat data, $\mathrm{RCN}$ is more active than POL. These interesting and meaningful differences between general rancimat and modified method may be due to changing of lipid composition which is suited to skin research.

In search of new antioxidant applicable to prevent skin lipid peroxidation, we selected meso-dihydroguaiaretic acid (MD$\mathrm{GA})^{8}$ as a candidate. MDGA, a naturally occurring polyphenol,

Table 2. Induction period and $P f$ value by modified rancimat method

\begin{tabular}{ccr}
\hline Compounds & Induction period $(\mathrm{h})^{a}$ & \multicolumn{1}{c}{$P f^{b}$} \\
\hline Control & 2.5 & 1.0 \\
BHT & 9.8 & 3.9 \\
SBG & 28.5 & 11.4 \\
POL & 5.5 & 2.2 \\
RCN & 10.0 & 4.0
\end{tabular}

${ }^{a}$ Values are given as means of three experiments. ${ }^{b} P f$ (protection factor) $=$ Induction period of antioxidant/Induction period of control. 


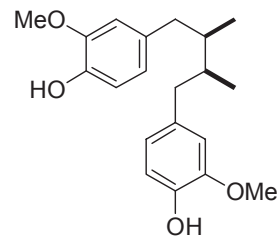

Figure 1. Structure of meso-dihydroguaiaretic acid (MDGA).

is considered a main antioxidative component of stems bark of Machilus thunbergii Sieb. Et Zucc (Fig. 1).

It showed broad biological activities such as hepatoprotective activity, ${ }^{8 \mathrm{a}}$ antibacterial activity ${ }^{8 \mathrm{~b}}$ inhibitory activity of MMP-2, 9 expression $^{8 \mathrm{c}}$ and inhibition of nitric oxide. ${ }^{8 \mathrm{~d}}$ We compared antioxidative activity of meso-dihydroguaiaretic acid (MDGA) with well known antioxidants such as L-ascorbic acid, $\alpha$-tocopherol and $\alpha$-lipoic acid using modified rancimat method. Among three known antioxidants, $\alpha$-tocopherol was found to be most active antioxidant. However, the activity of MDGA was more potent than that of $\alpha$-tocopherol. Activity order is MDGA $>\alpha$-tocopherol $>$ L-ascorbic acid $>\alpha$-lipoic acid.

In conclusion, we developed modified rancimat method to identify antioxidants to prevent peroxidation of skin lipid. Rancimat is the representative method to evaluate an antioxidative effect of various natural oils. The focus of rancimat assay is measurement of oil storage in food chemistry. Therefore this method is not fit in the case of skin lipid peroxidation. We used artificial skin lipid as a peroxidation lipid source and compared new modified method with general rancimat method by testing antioxidative activities of several natural extracts. Interestingly, activity order of tested natural products was changed and sensitivity of measuring antioxidative activity was dramatically increased. From these results, we evaluated meso-dihydroguaiaretic acid (MDGA) as a powerful natural antioxidant for skin lipid peroxidation using new rancimat method.

\section{Experimental Section}

Preparation of natural products and meso-dihydroguaiaretic acid (MDGA). Scutellaria baicalensis Georgi, Portulaca oleracea $\mathrm{L}$. and Rheum coreanum Nakaki were purchased from herbal market (Gunhwa Co.) in Suwon, Korea. meso-Dihydroguaiaretic acid (MDGA) was purchased from Elcom Science Co. in Seoul, Korea.

Preparation of natural product extracts. Crushed roots of Scutellaria baicalensis Georgi were ground into powder, and $500 \mathrm{~g}$ of the resulting powder was extracted three times, each time with $2 \mathrm{~L}$ of $70 \%$ ethanol, at room temperature. The extract was filtered through Whatman NO. 1 filter paper and concentrated to $200 \mathrm{~mL}$ in a rotary vacuum evaporator at $60{ }^{\circ} \mathrm{C}$. The yellow precipitate formed was filtered and dried. The aerial parts of Portulaca oleracea L. were dried in the shade and powdered. Whole dried Rheum coreanum Nakai was sliced and was ground into powder. Other steps are followed above mentioned.

General rancimat method. Antioxidative activities were studied with a Metrohm Rancimat Model 743 , at $100^{\circ} \mathrm{C}$. An automated Metrohm Rancimat apparatus model 743 (Metrohm, Switzerland) capable of operating over a temperature range of
Table 3. Oxidative stability of various antioxidants in modified rancimat method

\begin{tabular}{ccc}
\hline Compounds & ${\text { Induction period }(\mathrm{h})^{a}}^{a}$ & $\mathrm{PF}^{b}$ \\
\hline Control & 2.1 & 1.0 \\
BHT & 9.9 & 4.6 \\
L-Ascorbic acid & 6.6 & 3.1 \\
$\alpha$-Tocopherol & 17.3 & 8.1 \\
$\alpha$-Lipoic acid & 2.5 & 1.2 \\
MDGA & 29.0 & 13.6
\end{tabular}

${ }^{a}$ Values are given as means of three experiments. ${ }^{b} P f$ (protection factor) $=$ Induction period of antioxidant/Induction period of control.

$50-200{ }^{\circ} \mathrm{C}$ was used for induction period determination of samples. Samples were analyzed in the equipment at the same time. The air flow rate was fixed at $20 \mathrm{~L} / \mathrm{g}$. The concentration of test samples (SBG, POL and RCN), calculated on dry weight basis, was $0.25 \%$ level (w/w) including BHT in Table 1 and 2. Control means solvent oil used only. For oxidative stability measurement, each sample was weighted into the reaction vessel glassware. The conductimetry cells were filled with corn oil and deionized water up to $60 \mathrm{~mL}$. In Table 3, all tested samples (BHT, L-ascorbic acid, $\alpha$-tocopherol, $\alpha$-lipoic acid and MDGA) were evaluated at the $3 \mathrm{mM}$ concentration.

Modified rancimat method. We also prepared artificial skin lipid, consisting of pork lard as triglycerides (33\%), stearic acid as free fatty acids $(24 \%)$, lanolin as waxed $(22 \%)$, squalene $(12 \%)$ and cholesterol (4\%). The flow rate, temperature and concentration of samples were same as used in general rancimat method.

\section{References}

1. Kohen, R. Biomed. \& Pharmacother. 1999, 53, 181.

2. a) Meffert, H.; Diezel, W.; Sonnichsen, N. Experientia 1976, 32, 1397. b) Chamberlain, J.; Moss, S. H. Photochem. Photobiol. 1987, 45, 625. c) Niki, E. Free Radic. Biol. Med. 2009, 47, 469.

3. a) Morliere, P.; Moysan, A.; Tirache, I. Free Radic. Biol. Med. 1995, 19, 365. b) Polte, T.; Tyrrell, R. M. Free Radic. Biol. Med. 2004, 36, 1566.

4. a) Uchino, T.; Tokunaga, H.; Onodera, H.; Ando, M. Biol. Pharm. Bull. 2002, 25, 605. b) Ohsawa, K.; Watanabe, T.; Matsukawa, R.; Yoshimura, Y.; Imaeda, K. J. Toxicol. Sci. 1984, 9, 151. c) Kwon, T. W.; Olcott, H. S. J. Food Sci. 1966, 31, 552. d) Yeo, H. C. H.; Shibamoto, T. Lipids 1992, 27, 50. e) Uchino, T.; Tokunaga, H.; Ando, M. Toxicology In vitro 1999, 13, 483.

5. a) Chiba, K.; Sone, T.; Kawakami, K.; Onoue, M. Exp. Dermatol. 1999, 8, 471. b) Rho, H. S.; Baek, H. S.; Lee, B. S.; Kim, J. H.; Kim, D. H.; Chang, I. S. Bull. Korean. Chem. Soc. 2006, 27, 115.

6. a) Han, J.; Weng, X.; Bi, K. Food Chemistry 2008, 106, 2. b) Lee, J. H.; Lee, K. T.; Akoh, C. C.; Chung, S. K.; Kim, M. R. J. Agric. Food Chem. 2006, 54, 5416. c) Iqbal, S.; Bhanger, M. I. Food Chemistry 2007, 100, 246.

7. a) Clarys, P.; Barel, A. Clinics in Dermatology 1995, 13, 307. b) Musiał, W.; Kubis, A. Eur. J. Pharm. Biopharm. 2003, 55, 237.

8. a) Yu, Y. U.; Kang, S. Y.; Park, H. Y.; Sung, S. H.; Lee, E. J.; Kim, S. Y.; Kim, Y. C. J. Pharm. Pharmacol. 2000, 52, 1163. b) Karikome, H.; Mimaki, Y.; Sashida, Y. Phytochemistry 1991, 30, 315. c) Moon, H. I.; Chung, J. H. Biol. Pharm. Bull. 2005, 28, 2176. d) Ryu, J.-H.; Ahn, H.; Kim, J. Y.; Kim, Y.-K. Phytother. Res. 2003, 17, 485. e) Li, G.; Ju, H. K.; Chang, H. W.; Jahng, Y.; Lee, S.-H. Son, J.-K. Biol. Pharm. Bull. 2003, 26, 1039. 